\title{
AN "EXTRA" LAW FOR CHARACTERIZING MOUFANG LOOPS
}

\author{
ORIN CHEIN AND D. A. ROBINSON
}

\begin{abstract}
Let $(G, \cdot)$ be any loop and let $\lambda, \delta, \alpha$ be mappings of $G$ into $G$ so that $x \lambda=x \cdot x \delta=x(x \alpha \cdot x)$ for all $x \in G$. It is shown that the following conditions are equivalent: (a) $(x y \cdot z) x \alpha=x(y(z \cdot x \alpha))$ for all $x, y, z \in G,(\mathrm{~b})(G, \cdot)$ is Moufang and $x \delta$ is in the nucleus of $(G, \cdot)$ for all $x \in G,(\mathrm{c})(x y)(z \cdot x \lambda)=(x \cdot y z) x \lambda$ for ali $x, y, z \in G$. In particular, a loop $(G, \cdot)$ is extra in that $(x y \cdot z) x=x(y \cdot z x)$ for all $x, y, z \in G$ if and only if it satisfies the $M_{3}$-law in that $(x y)\left(z \cdot x^{3}\right)=$ $(x \cdot y z) x^{3}$ for all $x, y, z \in G$.
\end{abstract}

1. Introduction. F. Fenyves [3], [4] calls loops $(G, \cdot)$ with the property that $(x y \cdot z) x=x(y \cdot z x)$ for all $x, y, z \in G$ extra loops and shows that extra loops are necessarily Moufang. (Since commutative extra loops are associative, it is clear that there exist Moufang loops which are not extra. For constructions of extra loops see D. A. Robinson [6] and, in view of Corollary 2, see also $\mathrm{O}$. Chein and $\mathrm{H}$. Pflugfelder [2].) In this note we generalize Fenyves' result and at the same time provide what one might call an "extra" characterization of Moufang loops. Specifically, by virtue of our Theorem and Corollary 1 , a loop $(G, \cdot)$ is Moufang if and only if there is a mapping $\alpha$ of $G$ into $G$ so that

$$
(x y \cdot z) x \alpha=x(y(z \cdot x \alpha))
$$

for all $x, y, z \in G$. This particular result was announced by D. A. Robinson in a preliminary report (Notices Amer. Math. Soc. 18 (1971), Abstract 683-A10).

H. Pflugfelder [5] calls a loop $(G, \cdot)$ an $M$-loop provided that there is a mapping $\lambda$ of $G$ into $G$ such that

$$
(x y)(z \cdot x \hat{\lambda})=(x \cdot y z) x \lambda
$$

for all $x, y, z \in G$ and proves that $M$-loops are Moufang. More precisely,

Presented to the Society, November 20, 1971; received by the editors August 11, 1971. AMS 1970 subject classifications. Primary 20N05.

Key words and phrases. Extra loop, Moufang loop, $M$-loop, nucleus, inverse property, $M_{\lambda}$-loop.

(c) American Mathematical Society 1972 
she establishes what we shall refer to here as

PflugfeldeR's TheOREM. Let $(G, \cdot)$ be a loop whose nucleus is denoted by $N(G, \cdot)$ and let $\lambda$ and $\delta$ be mappings of $G$ into $G$ such that $x \lambda=x \cdot x \delta$ for all $x \in G$. Then (2) holds for all $x, y, z \in G$ if and only if $(G, \cdot)$ is Moufang and $x \delta \in N(G, \cdot)$ for all $x \in G$.

Furthermore, she calls (2) the $M_{\lambda}$-law for loops and also the $M_{k}$-law, where $k$ is a positive integer, provided that $\lambda$ is a power mapping of the form $\lambda: x \rightarrow x^{k}$. From Pflugfelder's Theorem it is evident that groups are precisely those Moufang loops which satisfy the $M_{2}$-law, a fact also mentioned in [2].

Our main purpose here is to clarify (see Theorem) the fundamental connection between the Pflugfelder identity (2) and our identity (1). We are then able to show (see Corollary 2) that extra loops are, in reality, exactly those Moufang loops for 'which the $M_{3}$-law holds.

2. Main result. In what follows $(G, \cdot)$ is a loop whose identity element is denoted by 1 and whose nucleus is denoted by $N(G, \cdot)$. For each $x \in G$ let $x J$ be that unique element in $G$ with the property that $x \cdot x J=1$. Clearly $J$ is a one-to-one mapping of $G$ onto $G$ so the inverse mapping $J^{-1}$ exists. It is easy to show that, for each $x \in G, x J^{-1}$ is that unique element in $G$ so that $x J^{-1} \cdot x=1$. For the most part we have adopted a terminology and a notation consistent with that of R. H. Bruck [1]. With this in mind we state and prove the following

THEOREM. Let $(G, \cdot)$ be a loop and let $\lambda, \delta, \alpha$ be mappings of $G$ into $G$ so that $x \lambda=x \cdot x \delta=x(x \alpha \cdot x)$ for all $x \in G$. Then the following conditions are equivalent:

(a) Identity (1) holds for all $x, y, z \in G$.

(b) $(G, \cdot)$ is Moufang and $x \delta \in N(G, \cdot)$ for all $x \in G$.

(c) Identity (2) holds for all $x, y, z \in G$.

Proof. The equivalence of (b) and (c) is immediate from Pflugfelder's Theorem.

(a) implies (b): Assume statement (a). Letting $x=y J^{-1}$ in (1), we see that $z \cdot y J^{-1} \alpha=y J^{-1}\left(y\left(z \cdot y J^{-1} \alpha\right)\right)$ for all $y, z \in G$. But, for each fixed $y \in G$, the mapping $z \rightarrow z \cdot y J^{-1} \alpha$ maps $G$ onto $G$ so, in fact, we have $z=y J^{-1} \cdot y z$ for all $y, z \in G$. Hence, $(G, \cdot)$ satisfies the left inverse property. Thus, $y J=$ $y J^{-1}(y \cdot y J)=y J^{-1}$ for all $y \in G$ so $J=J^{-1}$. Using this information and setting $z=y J$ in (1), we see that $(x y \cdot y J) x \alpha=x(y(y J \cdot x \alpha))=x \cdot x \alpha$ for all $x, y \in G$. Hence, $x y \cdot y J=x$ for all $x \in G$ and so $(G, \cdot)$ also satisfies the right inverse property. In other words, if (a) holds, then $(G, \cdot)$ is an inverse property loop. 
Now in (1) replace $z$ by $z \cdot x \alpha J$ to get

$$
((x y)(z \cdot x x J)) x \alpha=x\left(y\left(\left(z \cdot x \alpha_{J} J\right) \cdot x \alpha\right)\right)=x \cdot y z
$$

for all $x, y, z \in G$ and so by the inverse property we have $(x y)(z \cdot x \alpha J)=$ $(x \cdot y z) x \propto J$ for all $x, y, z \in G$. Hence, $(G, \cdot)$ satisfies the $M_{\theta}$-law where $\theta=\alpha J$. Then, by Pflugfelder's Theorem, $(G, \cdot)$ must be Moufang and $x \rho \in$ $N(G, \cdot)$ for all $x \in G$ where $x \rho$ is such that $x \theta=x \cdot x \rho$ for each $x \in G$. But since $(G, \cdot)$ is an inverse property loop it follows (see Bruck $[1$, p. 111]) that $(x y) J=y J \cdot x J$ for all $x, y \in G$ and so $x \rho J=x \theta J \cdot x=x \alpha \cdot x$ for all $x \in G$. Hence, $(G, \cdot)$ is Moufang and $x \delta=x \alpha \cdot x \in N(G, \cdot)$ for all $x \in G$. Thus, (b) holds.

(b) implies (a): Assume statement (b). Since $(G, \cdot)$ is then Moufang, the inverse property holds (see Bruck [1, p. 115]) and so once again $(x y) J=$ $y J \cdot x J$ for all $x, y \in G$. Now, just as above, let $\theta=\alpha J$. But $x \delta=x \alpha \cdot x \in N(G, \cdot)$ for all $x \in G$ implies that $x J \cdot x \theta=x J \cdot x \alpha J=(x \alpha \cdot x) J \in N(G, \cdot)$ for all $x \in G$. Hence, Pflugfelder's Theorem is applicable and allows us to conclude that the $M_{\theta}$-law holds. Replacing $z$ by $z \cdot x \alpha$ in the $M_{\theta}$-law, we see that

$$
(x y)((z \cdot x \alpha) x \theta)=(x(y(z \cdot x \alpha))) x \theta
$$

for all $x, y, z \in G$. This last observation, in conjunction with the inverse property, indicates that

$$
\begin{aligned}
x y \cdot z & =(x y)((z \cdot x \alpha) x x J)=(x y)((z \cdot x \alpha) x \theta) \\
& =(x(y(z \cdot x \alpha))) x \theta \\
& =(x(y(z \cdot x \alpha))) x \alpha J \text { for all } x, y, z \in G .
\end{aligned}
$$

One final appeal to the inverse property now yields $(x y \cdot z) x \alpha=x(y(z \cdot x \alpha))$ for all $x, y, z \in G$. Hence, (a) holds and the proof is complete.

The following applications of the preceding theorem are of interest.

Corollary 1. A loop $(G, \cdot)$ is Moufang if and only if

$$
(x y \cdot z) x J=x(y(z \cdot x J))
$$

for all $x, y, z \in G$.

Proof. This follows directly from the Theorem by choosing $x \alpha=x J$ and $x \lambda=x(x J \cdot x)=x$ for all $x \in G$. (The last equality holds since each of conditions (a) and (c) of the Theorem implies that $(G, \cdot)$ is Moufang and, hence, that $x J \cdot x=1$ for all $x \in G$.)

COROLLARY 2. For any loop $(G, \cdot)$ the following conditions are equivalent:

(a') $(G, \cdot)$ is an extra loop.

(b') $(G, \cdot)$ is Moufang and $x^{2} \in N(G, \cdot)$ for all $x \in G$.

(c') The $M_{3}$-law holds for $(G, \cdot)$. 
Proof. This is also an immediate consequence of the theorem for the special case $x \alpha=x, x \delta=x \cdot x=x^{2}$, and $x \lambda=x \cdot x^{2}=x^{3}$ for all $x \in G$. (Note that the equivalence of $\left(\mathrm{a}^{\prime}\right)$ and $\left(\mathrm{b}^{\prime}\right)$ has also been proved by Fenyves $[\mathbf{4}$, Theorem 1].)

\section{REFERENCES}

1. R. H. Bruck, A survey of binary systems, Ergebnisse der Mathematik und ihrer Grenzgebiete, Neue Folge, Heft 20, Reihe: Gruppentheorie, Springer-Verlag, Berlin and New York, 1958. MR 20 \#76.

2. O. Chein and H. Pflugfelder, On maps $x \rightarrow x^{n}$ and the isotopy-isomorphy property of Moufang loops, Aequationes Math. 6 (1971), 157-161.

3. F. Fenyves, Extra loops. I, Publ. Math. Debrecen 15 (1968), 235-238. MR 38 \#5976.

4. - Extra loops. II. On loops with identities of Bol-Moufang type, Publ. Math. Debrecen 16 (1969), 187-192. MR 41 \#7017.

5. H. Orlik-Pflugfelder, A special class of Moufang loops, Proc. Amer. Math. Soc. 26 (1970), 583-586. MR 42 \#407.

6. D. A. Robinson, Holomorphy theory of extra loops, Publ. Math. Debrecen (to appear).

Department of Mathematics, Temple University, Philadelphia, Pennsylvania 19122

School of Mathematics, Georgia institute of Technology, Atlanta, GeORGIA 30332 